\title{
IL35 Attenuated LPS-Induced Acute Lung Injury by Regulating Macrophage Polarization
}

Shengsong Chen ( $\nabla$ chenss93@163.com )

China-Japan Friendship Hospital https://orcid.org/0000-0003-2763-943X

Jingen Xia

China-Japan Friendship Hospital

Yi Zhang

China-Japan Friendship Hospital

Qingyuan Zhan

China-Japan Friendship Hospital

\section{Research Article}

Keywords: Interleukin 35, Acute lung injury, Inflammation, Macrophage polarization

Posted Date: January 3rd, 2022

DOI: https://doi.org/10.21203/rs.3.rs-958085/v2

License: (c) (1) This work is licensed under a Creative Commons Attribution 4.0 International License. Read Full License 


\section{Abstract}

Background Interleukin 35 (IL35) has been reported to play a role in acute lung injury (ALI); however, the current results on the relationship between IL35 and ALI are inconsistent. Therefore, we will further determine the function of IL35 in mouse ALI and its potential mechanism in this paper.

Materials and Methods HE staining and Masson staining were used to evaluate lung injury in mice. Immunohistochemical staining was used to calculate the expression of IL35 p35, TLR4 and MD2 and the ratio of $\mathrm{Bax} / \mathrm{Bcl} 2$ and p-P65/P65. The expression levels of IL35 EBi3, CD68, CD206 and MPO were detected by immunofluorescence staining. RT-PCR was used to examine the expression levels of IL1 $\beta$ and IL6. TUNEL staining was performed to detect apoptotic cells.

Results Overexpression of IL35 alleviated LPS-induced acute lung injury in mice. IL35 overexpression decreased the expression of CD68 and increased the expression of CD206 in ALI mice. Furthermore, upregulation of IL35 expression obviously reduced the expression of MPO, IL1 $\beta$ and IL6 in lung tissues of mice with ALI. Mechanistically, IL35 suppressed the TLR4/NFKB-P65 pathway, leading to the promotion of $\mathrm{M} 1$ to $\mathrm{M} 2$ macrophage transition and inflammation relief in ALI in mice.

Conclusions IL35 relieved LPS-reduced inflammation and ALI in mice by regulating M1/M2 macrophage polarization and inhibiting the activation of the TLR4/NFKB-P65 pathway.

\section{Introduction}

Acute lung injury (ALI) is characterized by alveolar and capillary endothelial damage and a permeability increase in the alveolar-capillary membrane, which are noncardiac pathogenic causes that progress to hypoxic respiratory failure and even acute respiratory distress syndrome (ARDS) and death[1]. The main treatments for ALI/ARDS are antibacterial drugs, corticosteroids, lung protective ventilation strategy (LPVS) and extracorporeal membrane oxygenation (ECMO). Unfortunately, all of these approaches have some problems, particularly no benefits in some cases or side effects or high costs, and may still result in high morbidity and mortality of ALI/ARDS [2,3]. Therefore, it is urgent to explore and elucidate the physiological and molecular mechanisms of ALI/ARDS.

An increasing number of studies have confirmed that inflammation plays a crucial role in the initiation and development of ALI[4, 5]. With the increase in proinflammation and the decrease in the antiinflammatory response in ALI, a large number of inflammatory cytokines, such as IL6 and TNF-a, are secreted, and cytokine storms form, resulting in respiratory failure and ARDS[6]. Macrophage activation is considered to be the critical initial stage of the inflammatory response[7, 8]. Macrophages have high plasticity and can polarize into distinct functional phenotypes depending on different stimuli[7]. In particular, many studies have found that macrophages are polarized to the M1 phenotype (proinflammatory effect) rather than the M2 phenotype (anti-inflammatory/reparative effect) in ALI, leading to aggravated lung inflammation $[9,10]$. Therefore, accelerated M1/M2 macrophage switching is the key to improving lung inflammatory damage. 
Interleukin 35 (IL35) is a newly identified cytokine coming from the IL12 heterodimeric cytokine family composed of Epstein-Barr virus-induced gene 3 (EBi3) and the IL12 p35 subunit (IL-12A)[11, 12]. IL35 is primarily secreted by $T$ cells and $B$ cells and plays an important role in inflammatory and immune responses[11, 13]. In recent years, a growing number of studies have provided insight into the relationship between IL35 and pulmonary inflammatory diseases and found that IL35 could protect the lung from inflammation and autoimmune diseases, including asthma and respiratory infection[14, 15]. However, the results on the role of IL35 in ALI/ARDS are inconsistent. Cao observed that IL35 was elevated in sepsis and mediated inflammation[16]. Pan and his colleagues reported that IL35 was downregulated in ALI and that overexpression of IL35 reduced inflammation[17]. Wang and his colleagues showed that IL35 was upregulated and improved endothelial injury in ALI[18]. In addition, whether IL35 regulates macrophage polarization in ALI remains unclear. Thus, we further explicated the role of IL35 and explored whether IL35 had an effect by regulating macrophage polarization in ALI.

In this study, we observed the expression and function of IL35 in an in vivo model of mouse ALI. Next, we detected the markers of $\mathrm{M} 1$ and $\mathrm{M} 2$ macrophages using immunofluorescence staining to determine the potential mechanism of IL35 in ALI. Our findings may provide a novel basis for the development of therapies targeting ALI/ARDS.

\section{Materials And Methods}

\section{Animal studies}

Male C57BL/ 6 mice (aged 8-10 weeks, body weight $22 \pm 3 \mathrm{~g}$ ) were purchased from Beijing SiPeiFu Biotechnology Co., Ltd, China. All mice were housed conventionally at $22^{\circ} \mathrm{C}$ under a $12-\mathrm{h}$ light-dark cycle with access to water and food ad libitum. All animal experiments were performed in compliance with the National Institutes of Health $(\mathrm{NIH})$ policies in the Guide for the Care and Use of Laboratory Animals and were approved by the Experimental Animal Ethics Committee of CHINA-JAPAN Friendship Hospital.

Eighteen mice were randomly divided into three groups of 6 mice each, including a control group, an acute lung injury (ALI) group and an ALI+ plasmid DNA containing the IL35 gene (pcDNA-IL35) group. pcDNA-IL35 was purchased from Gene Pharma (Shanghai, China) and injected into mice by tail intravenous injection (i.v.) according to the manufacturer's instructions[19]. The experimental procedures are described below (Fig. 1). Mice from the control group were given $2 \mathrm{ml}$ of PBS by i.v. and received an intraperitoneal injection (i.p.) of normal saline after 7 days. Mice from the ALI group were given $2 \mathrm{ml}$ of PBS by i.v. and received an i.p. injection of LPS (10 mg/ $\mathrm{kg}$, Sigma, USA) after 7 days. Mice from the ALI+IL35 group were given $2 \mathrm{ml}$ of PBS containing $100 \mu \mathrm{g}$ of pcDNA-IL35 by i.v. and received an i.p. injection of LPS (10 mg/kg) after 7 days. All mice were anesthetized intraperitoneally with an overdose of sodium pentobarbital at $12 \mathrm{~h}$ postinjury. Subsequently, the lungs were perfused and isolated for collection of paraffin sections for histological examination and PCR analyses.

\section{Hematoxylin-eosin (HE) staining}


Lung tissues were collected and fixed in $4 \%$ paraformaldehyde. Then, $5-\mu \mathrm{m}$ sections from the paraffin blocks of tissues were stained with hematoxylin-eosin (HE) and observed under an optical microscope (Olympus, Japan). The lung injury score was calculated by two researchers without group information as described previously[20]. Five independent variables, including neutrophils in the alveolar space, neutrophils in the interstitial space, the existence of hyaline membranes, proteinaceous debris filling the airspaces and alveolar septal thickening, were used to generate a lung injury score[20].

\section{Masson staining}

Lung tissues were stained with a Ponceau-fuchsin solution, rinsed with glacial acetic acid and then immersed in phosphomolybdic acid. After the excess liquid was absorbed by the filter paper, the sections were stained with an aniline blue solution, soaked in glacial acetic acid, washed with water, air-dried, treated with xylene three times and then mounted. An optical microscope (Olympus, Japan) was used to detect collagen deposition appearing on the airway walls of lung tissues.

\section{Immunofluorescence and immunohistochemical staining}

Lung tissues were collected and fixed in $4 \%$ paraformaldehyde, and immunofluorescence staining was performed according to the manufacturer's standard protocol[8]. Briefly, tissue specimens were cut into 5$\mu \mathrm{m}$ serial sections and blocked with $10 \%$ blocking serum in PBS. The sections were then incubated with primary antibodies against IL35 EBi3 (Abclonal, A19613, China) and CD68 (Abcam, ab53444, USA) or CD206 (Santa Cruz, sc-58986, USA), MPO (Abcam, ab208670, USA) at $4^{\circ} \mathrm{C}$ overnight. Next, the sections were incubated with Alexa Fluor 488-conjugated goat anti-rabbit IgG secondary antibody or Alexa Fluor Plus 555-conjugated goat anti-rabbit IgG secondary antibody or Alexa Fluor 488 -conjugated goat anti-rat IgG secondary antibody or Alexa Fluor 488 -conjugated goat anti-mouse IgG secondary antibody (Molecular Probes, Invitrogen, USA) for 1 hour at room temperature. DAPI (Invitrogen, USA) was used for nuclear staining. The sections were observed with a fluorescence microscope (Olympus, Japan), and the images were analyzed using ImageJ (Bethesda, MD, USA).

Immunohistochemical staining was used to evaluate IL35 p35, Bax, Bcl2, TLR4, MD2 and p-P65/P65 in lung tissues according to the manufacturer's standard protocol[21]. Briefly, tissue specimens were cut into 5 - $\mu \mathrm{m}$ serial sections, deparaffinized, rehydrated, blocked and incubated with primary antibodies against IL35 p35 (R\&D Systems, MAB6688, USA), Bax (Abcam, ab32503, USA), Bcl2 (Abcam, ab182858, USA), TLR4 (Abcam, ab13867, USA), MD2 (Abcam, ab24182, USA), p-P65 (Abclonal, AP0475, China) or P65 (Abcam, ab16502, USA) at $4^{\circ} \mathrm{C}$ overnight. Next, the sections were incubated with biotinylated IgG (1:250) for 1 hour and then with streptavidin-HRP for $30 \mathrm{~min}$ at room temperature. DAB was then added to each section for $5 \mathrm{~min}$. The sections were observed with a microscope (Olympus, Japan), and the images were analyzed using Image-Pro Plus 6.0 (Media cybernetics, USA).

\section{TUNEL staining}

Lung tissues were collected and fixed in $4 \%$ paraformaldehyde, and tissue specimens were cut into $5-\mu m$ serial sections. Terminal deoxynucleotide transferase-mediated dUTP nick end-labeling (TUNEL) staining 
was performed using an In Situ Cell Death Detection Kit (Roche, Mannheim, Germany) following the manufacturer's instructions. The sections were observed with a fluorescence microscope (Olympus, Japan) with 10 fields of view randomly selected, and the rate of cell apoptosis was determined using ImageJ (Bethesda, MD, USA). Green nuclei were considered positive-apoptotic cells. The cells with blue nuclei were deemed to be normal, and the average value was determined accordingly. The ratio of the number of green cells to that of blue cells was regarded as the rate of cell apoptosis.

\section{Quantitative real-time PCR}

Total RNA was extracted from lung tissues using TRIzol reagent (Invitrogen Life Technologies, Carlsbad, $\mathrm{CA}$ ), and cDNA synthesis was performed using random hexamer primers and a reverse transcription kit (TaKaRa, Japan) according to the manufacturer's protocol[11]. RT-PCR was performed using a SYBRß PremixEx Taq II Kit (TaKaRa, Japan) on a 7500 Fast Real Time PCR system from Applied Biosystems (Bio-Rad, USA). The relative gene expression levels were determined by the comparative critical threshold $(\triangle \Delta \mathrm{CT})$ method. Primer sequences of mouse genes were in Table 1.

Table 1

Primer sequences used for RT-PCR analysis

\begin{tabular}{|lll|}
\hline Genes & \multicolumn{2}{|c|}{ Sequences } \\
\hline IL1 $\beta$ & forward & 5'-GCATCCAGCTTCAAATCTCGC-3' \\
& reverse & 5'-TGTTCATCTCGGAGCCTGTAGTG-3' \\
IL6 & forward & 5'-AGTTGCCTTCTTGGGACTG-3' \\
& reverse & 5'-AGGTCTGTTGGGAGTGGTATC-3' \\
GAPDH & forward & 5'-AAGAAGGTGGTGAAGCAGGCATC-3' \\
& reverse & 5'-CGGCATCGAAGGTGGAAGAGTG-3' \\
\hline
\end{tabular}

\section{Statistical analysis}

Data are presented as the means \pm S.E.M. One-way ANOVA with Dunnett or Bonferroni tests was used to analyze statistical significance as appropriate. $P<0.05$ was considered statistically significant. The statistical analyses were performed using Statistical Product and Service Solutions 19.0 (SPSS, Systat Software, San Jose, CA, USA).

\section{Results}

\section{IL35 alleviates LPS-induced acute lung injury}

IL35 has been reported to play immunosuppressive and anti-inflammatory roles in infections, inflammation, and autoimmune diseases[11]. We examined whether IL35 alleviated LPS-induced ALI in our study. The expression of IL35 p35 was first detected by immunohistochemical staining. The results 
showed that LPS-induced ALI promoted IL35 p35 up regulation and the expression of IL35 p35 was further overexpression after pcDNA-IL35 transfection $(P<0.05$, Fig. 2A-B). HE staining and lung injury scores were then used to evaluate the severity of lung injury. As shown in Fig. 2C, LPS treatment increased alveolar collapse and thickened the alveolar wall and septa; however, overexpression of IL35 obviously reduced LPS-stimulated lung tissue injury ( $P<0.05$, Fig. 2c). A similar trend appeared in the lung injury score $(P<0.05$, Fig. 2D). We next detected collagen deposition in lung tissues by Masson staining. As expected, LPS increased collagen deposition in lung tissues, and upregulation of IL35 inhibited collagen deposition in ALI $(P<0.05$, Fig. 2E-F). The above data suggested that IL35 may alleviate LPS-induced acute lung injury.

\section{IL35 regulates macrophage polarization in ALI in mice}

Some studies have found that IL35 ameliorates tissue injury by stimulating macrophage polarization[22, 23]. Therefore, we hypothesized that IL35 reduced ALI by regulating macrophage polarization. To explore whether IL35 decreased the levels of M1 macrophages and increased the levels of M2 macrophages in ALI, we detected the expression of IL35 EBi3, the other subunit of IL35, and markers of M1/M2 macrophages (CD68 and CD206) by immunofluorescence staining in lung tissues. The results showed that IL35 overexpression led to a decrease in the levels of CD68 and an increase in the levels of CD206 in ALI in mice $(P<0.05$, Fig. 3A-E).

\section{IL35 mitigates inflammation in ALI in mice}

The imbalance between the activation and regulation of macrophage polarization may accelerate the development of ALI/ARDS[10,24]. We observed that IL35 may regulate macrophage polarization in ALI in mice. We then explored whether IL35 reduced inflammation in ALI. As expected, the expression levels of MPO protein in lung tissues of mice with ALI were found to be higher than those in the ALI+ pcDNA-IL35 group ( $P<0.05$, Fig. 4A-B). RT-PCR was used to check the levels of inflammatory cytokines. Similarly, the levels of IL1 $\beta$ and IL6 were decreased in the lung tissues from the ALI+ pcDNA-IL35 group compared with those in the ALI group $(P<0.05$, Fig. 4C-D). These results demonstrated that IL35 may mitigate inflammation by regulating macrophage polarization.

\section{IL35 lessens apoptosis in ALI in mice}

To further confirm whether IL35 improved lung injury, TUNEL staining and immunohistochemical staining were used to assess apoptosis in lung tissues of mice with ALI. The results showed that IL35 overexpression decreased the rate of cell apoptosis induced by LPS $(P<0.05$, Fig. 5A-B). Consistently, upregulation of IL35 reduced the rate of $\mathrm{Bax} / \mathrm{Bc} 22$ in the lung tissues of mice, which was high after LPS treatment $(P<0.05$, Fig. 5C-D).

\section{IL35 regulates macrophage polarization by the TLR4/NFKB- P65 pathway}


A compelling body of evidence has shown that the TLR4/NFKB-P65 pathway is a critical signal involved in macrophage polarization[10, 25, 26]. However, whether IL35 regulates macrophage polarization in ALI by inhibiting the TLR4/NFKB-P65 pathway remains unclear. To gain insight into the mechanism by which IL35 improved ALI, we detected the expression of TLR4, MD2 or p-P65/P65 by immunohistochemical staining. High levels of TLR4 and MD2 expression were observed in ALI in mice, but IL35 overexpression stopped them from rising $(P<0.05$, Fig. 6A-D). The same trend was observed for the rate of p-P65/P65 expression ( $P<0.05$, Fig. $6 \mathrm{E}-\mathrm{F})$. Furthermore, we found that the expression of TLR4 and MD2 and the rate of p-P65/P65 were also higher in ALI group than in control group in pulmonary macrophages, overexpression of IL35 prevented them from increasing in pulmonary macrophages of ALI. All these results suggested that IL35 may regulate macrophage polarization by the TLR4/NFKB-P65 pathway in LPS-induced ALI.

\section{Discussion}

ALI/ARDS is an acute and life-threatening pulmonary inflammatory disease with a high mortality rate of up to $40-60 \%[10,27]$. An imbalance in macrophage polarization is crucial to initiate the inflammatory response in ALI. IL35 is a novel cytokine from the IL12 family and has been reported to reduce the progression of some inflammatory diseases [11-13]. Here, we observed that the expression of IL35 in lung tissues was upregulated in ALI in mice and that IL35 overexpression promoted the transformation of M1 to M2 macrophages and relieved inflammation, resulting in improved LPS-induced ALI in mice. Our results and previous studies consistently suggested that IL35 has a protective effect and may be a key therapeutic target in ALI/ARDS in the future $[17,18]$.

Accumulating evidence has shown that IL35 may be produced by T cells, dendritic cells (DCs), B cells and even nonimmune cells [28-30]. However, the constitutive nature of normal tissues is not a characteristic of IL35 [28]. The expression of IL35 is low or even not examined in some immune cells and can be triggered under inflammatory conditions [28,31]. Thus, IL35 constitutes an important mediator of inflammation and contributes to regulating inflammatory disease progression. For example, Li et al. confirmed that IL35 suppressed allergic airway inflammation in asthma [15]. Hu suggested that IL35 alleviates LPS-induced acute kidney injury in mice by inhibiting proinflammatory cytokine production [19]. Pan and his colleagues reported that IL35 protected against cigarette smoke-induced lung inflammation in mice [11]. In addition, researchers have recently gained insight into the role of IL35 in ALI/ARDS. Our study and former reports agreed that overexpression of IL35 reduced inflammation and improved ALI[17, 18]. However, Ju and his colleagues observed lower bacterial loads after treating septic mice with antiIL35 p35 antibodies [16]. This is in line with a previous report that IL23 p35 knockout enhanced the acute immune response against Staphylococcus aureus infection [32]. IL12 p35 is a mutant subunit of IL35 and regulates the activity of IL35 $[11,12,16]$. Ye et al. observed that there was obviously different expression of IL12 p35 in macrophages, B lymphocytes, T lymphocytes and DCs after stimulation by harmful substances [13]. The differential expression of IL12 p35 in different cells may represent different roles in inflammatory diseases. The conflicting findings that IL35 mediates or reduces inflammation in ALI suggest that more research is needed to confirm the current results and potential mechanism[16-18]. 
$\mathrm{M} 1 / \mathrm{M} 2$ macrophage polarization contributes to the development of ALI[8,33]. A growing amount of data verified that the M1/M2 macrophage profile toward M1 leads to aggravation and deterioration of ALI/ARDS[8, 33, 34]. Li suggested that HSF1 attenuates LPS-induced ALI by suppressing macrophage infiltration and CCR2 expression[8]. Cui found that IncRNA Malat1 knockdown attenuated LPS-induced M1 macrophage activation and enhanced M2 differentiation, resulting in diminished lung injury [33]. Zhang showed that MCP-induced protein 1 attenuated sepsis-induced ALI by decreasing the M1/M2 macrophage ratio[34]. In this study, our results showed that IL35 overexpression decreased the expression of the M1 phenotype-specific marker CD68 and increased the expression of the M2 phenotype-specific marker CD206, leading to relief of inflammation and pulmonary injury in ALI mice. Our data are in line with previous results showing that overexpression of IL35 protects pulmonary tissue from LPS-induced ALI by promoting M1 to M2 macrophage polarization.

We further explored how IL35 regulated macrophage polarization to reduce pulmonary inflammatory damage in mice with ALI. LPS is an important component of gram-negative bacteria and a ligand of TLR4[25]. TLR4, an important transmembrane pattern-recognition receptor of the innate immune system, is recruited and activated after LPS stimulation, leading to the initiation of the NFKB-P65 signaling pathway and the release of proinflammatory cytokines, such as IL1 $\beta$ and IL6[26, 35]. In our study, upregulation of IL35 obviously suppressed the expression of TLR4 and MD2, as well as p-P65/P65. This trend also existed in pulmonary macrophages of ALI. Previous studies have shown that IL35 responds to TLR4 agonists [30]. Thus, we suspected that IL35 may interact with TLR4 to inhibit the TLR4/NFKB-P65 pathway, reducing inflammatory lung injury. More studies are needed to further confirm the underlying mechanism in the future.

In conclusion, IL35 relieved LPS-reduced inflammation and ALI in mice by regulating M1/M2 macrophage polarization and inhibiting the activation of the TLR4/NFKB-P65 pathway (Fig. 7). Our findings may provide new insight into ALI/ARDS treatment by targeting the regulation of IL35 expression.

\section{Declarations}

Author Contributions Conception and design: Sheng-Song Chen, Jin-Gen Xia, Yi Zhang, Qing-Yuan Zhan; Conduct experiment: Sheng-Song Chen, Jin-Gen Xia; Data analysis: Sheng-Song Chen, Yi Zhang; Drafting manuscript: Sheng-Song Chen, Qing-Yuan Zhan; Interpretation and revised manuscript: Sheng-Song Chen, Jin-Gen Xia, Yi Zhang, Qing-Yuan Zhan.

Funding This work was supported by the National Natural Science Foundation of China (81870072).

Data availability The datasets supporting the conclusions of this article are included within the article.

Conflict of interest All the authors declare no confict of interest.

Compliance with Ethical Standards All applicable CHINA-JAPAN Friendship Hospital guidelines for the care and use of animals were followed. 


\section{References}

1. Wheeler AP, Bernard GR (2007) Acute lung injury and the acute respiratory distress syndrome: a clinical review. Lancet 369:1553-1564. doi: 10.1016/S0140-6736(07)60604-7

2. Hotchkiss RS, Monneret G, Payen D (2013) Immunosuppression in sepsis: a novel understanding of the disorder and a new therapeutic approach. Lancet Infect Dis 13:260-268. doi: 10.1016/S14733099(13)70001-X

3. Wenzel RP, Edmond MB (2012) Septic shock-evaluating another failed treatment. N Engl J Med 366:2122-2124. doi: 10.1056/NEJMe1203412

4. Fowler AA 3, Truwit JD, Hite RD, Morris PE, DeWilde C, Priday A, Fisher B, Thacker LR 2, Natarajan R, Brophy DF, Sculthorpe R, Nanchal R, Syed A, Sturgill J, Martin GS, Sevransky J, Kashiouris M, Hamman S, Egan KF, Hastings A, Spencer W, Tench S, Mehkri O, Bindas J, Duggal A, Graf J, Zellner S, Yanny L, McPolin C, Hollrith T, Kramer D, Ojielo C, Damm T, Cassity E, Wieliczko A, Halquist M (2019) Effect of Vitamin C Infusion on Organ Failure and Biomarkers of Inflammation and Vascular Injury in Patients With Sepsis and Severe Acute Respiratory Failure: The CITRIS-ALI Randomized Clinical Trial. JAMA 322:1261-1270. doi: 10.1001/jama.2019.11825

5. Tong Y, Yu Z, Chen Z, Zhang R, Ding X, Yang X, Niu X, Li M, Zhang L, Billiar TR, Pitt BR, Li Q (2021) The HIV protease inhibitor Saquinavir attenuates sepsis-induced acute lung injury and promotes M2 macrophage polarization via targeting matrix metalloproteinase-9. Cell Death Dis 12:67. doi: 10.1038/s41419-020-03320-0

6. Zhang X, Zhang Z, Ju M, Li J, Jing Y, Zhao Y, Gu C, Dong M, Li G, Liu Y (2020) Pretreatment with interleukin 35-engineered mesenchymal stem cells protected against lipopolysaccharide-induced acute lung injury via pulmonary inflammation suppression. Inflammopharmacology 28:1269-1281. doi: 10.1007/s10787-020-00696-5

7. Syed MA, Bhandari V (2013) Hyperoxia exacerbates postnatal inflammation-induced lung injury in neonatal BRP-39 null mutant mice promoting the M1 macrophage phenotype. Mediators Inflamm 2013:457189. doi: $10.1155 / 2013 / 457189$

8. Li T, Xiao G, Tan S, Shi X, Yin L, Tan C, Gu J, Liu Y, Deng H, Liu K, Liu M, Zhang H, Xiao X (2020) HSF1 Attenuates LPS-Induced Acute Lung Injury in Mice by Suppressing Macrophage Infiltration. Oxid Med Cell Longev 2020:1936580. doi: 10.1155/2020/1936580

9. Kumar V (2020) Pulmonary Innate Immune Response Determines the Outcome of Inflammation During Pneumonia and Sepsis-Associated Acute Lung Injury. Front Immunol 11:1722. doi: 10.3389/fimmu.2020.01722

10. Wang L, Zhang H, Sun L, Gao W, Xiong Y, Ma A, Liu X, Shen L, Li Q, Yang H (2020) Manipulation of macrophage polarization by peptide-coated gold nanoparticles and its protective effects on acute lung injury. J Nanobiotechnology 18:38. doi: 10.1186/s12951-020-00593-7

11. Pan X, Xu K, Li Y, Wang X, Peng X, Li M, Li Y (2019) Interleukin-35 expression protects against cigarette smoke-induced lung inflammation in mice. Biomed Pharmacother 110:727-732. doi: 
10.1016/j.biopha.2018.12.028

12. Ye J, Huang Y, Que B, Chang C, Liu W, Hu H, Liu L, Shi Y, Wang Y, Wang M, Zeng T, Zhen W, Xu Y, Shi L, Liu J, Jiang H, Ye D, Lin Y, Wan J, Ji Q (2018) Interleukin-12p35 Knock Out Aggravates DoxorubicinInduced Cardiac Injury and Dysfunction by Aggravating the Inflammatory Response, Oxidative Stress, Apoptosis and Autophagy in Mice. EBioMedicine 35:29-39. doi:

10.1016/j.ebiom.2018.06.009

13. Ye J, Que B, Huang Y, Lin Y, Chen J, Liu L, Shi Y, Wang Y, Wang M, Zeng T, Wang Z, Hu H, Xu Y, Shi L, Ye D, Liu J, Jiang H, Wan J, Ji Q (2019) Interleukin-12p35 knockout promotes macrophage differentiation, aggravates vascular dysfunction, and elevates blood pressure in angiotensin IIinfused mice. Cardiovasc Res 115:1102-1113. doi: 10.1093/cvr/cvy263

14. Branchett WJ, Lloyd CM (2019) Regulatory cytokine function in the respiratory tract. Mucosal Immunol 12:589-600. doi: 10.1038/s41385-019-0158-0

15. Li Y, Pan X, Peng X, Li S, Zhou Y, Zheng X, Li M (2015) Adenovirus-mediated interleukin-35 gene transfer suppresses allergic airway inflammation in a murine model of asthma. Inflamm Res 64:767-774. doi: 10.1007/s00011-015-0858-1

16. Cao J, Xu F, Lin S, Tao X, Xiang Y, Lai X, Zhang L (2015) IL-35 is elevated in clinical and experimental sepsis and mediates inflammation. Clin Immunol 161:89-95. doi: 10.1016/j.clim.2015.08.016

17. Pan $W, X u X$, Wang $Y$, Song $X$ (2020) Interleukin-35 reduces inflammation in acute lung injury through inhibiting TLR4/NF-kappaB signaling pathways. Exp Ther Med 19:1695-1700. doi: 10.3892/etm.2020.8407

18. Sha X, Meng S, Li X, Xi H, Maddaloni M, Pascual DW, Shan H, Jiang X, Wang H, Yang XF (2015) Interleukin-35 Inhibits Endothelial Cell Activation by Suppressing MAPK-AP-1 Pathway. J Biol Chem 290:19307-19318. doi: 10.1074/jbc.M115.663286

19. Hu L, Chen C, Zhang J, Wu K, Zhang X, Liu H, Hou J (2017) IL-35 Pretreatment Alleviates Lipopolysaccharide-Induced Acute Kidney Injury in Mice by Inhibiting NF-kappaB Activation. Inflammation 40:1393-1400. doi: 10.1007/s10753-017-0582-9

20. Matute-Bello G, Downey G, Moore BB, Groshong SD, Matthay MA, Slutsky AS, Kuebler WM, Acute Lung Injury in Animals Study G (2011) An official American Thoracic Society workshop report: features and measurements of experimental acute lung injury in animals. Am J Respir Cell Mol Biol 44:725-738. doi: 10.1165/rcmb.2009-0210ST

21. Yu LL, Wang Y, Xiao ZK, Chen SS (2021) Heat shock protein B8 promotes proliferation and migration in lung adenocarcinoma $A 549$ cells by maintaining mitochondrial function. Mol Cell Biochem 476:187-197. doi: 10.1007/s11010-020-03896-3

22. Peng M, Qiang L, Xu Y, Li C, Li T, Wang J (2019) IL-35 ameliorates collagen-induced arthritis by promoting TNF-alpha-induced apoptosis of synovial fibroblasts and stimulating M2 macrophages polarization. FEBS J 286:1972-1985. doi: 10.1111/febs.14801

23. Liu X, Zhang R, Hou J, Wu J, Zhang M, Fang S, Wang X, Huang X, Tian J, Li H, Sun Y, Yu B (2019) Interleukin-35 promotes early endothelialization after stent implantation by regulating macrophage 
activation. Clin Sci (Lond) 133:869-884. doi: 10.1042/CS20180879

24. Tu GW, Shi Y, Zheng YJ, Ju MJ, He HY, Ma GG, Hao GW, Luo Z (2017) Glucocorticoid attenuates acute lung injury through induction of type 2 macrophage. J Transl Med 15:181. doi: 10.1186/s12967-0171284-7

25. Pinheiro NM, Santana FP, Almeida RR, Guerreiro M, Martins MA, Caperuto LC, Camara NO, Wensing LA, Prado VF, Tiberio IF, Prado MA, Prado CM (2017) Acute lung injury is reduced by the alpha7nAChR agonist PNU-282987 through changes in the macrophage profile. FASEB J 31:320332. doi: 10.1096/fj.201600431R

26. Vergadi E, Vaporidi K, Theodorakis EE, Doxaki C, Lagoudaki E, leronymaki E, Alexaki VI, Helms M, Kondili E, Soennichsen B, Stathopoulos EN, Margioris AN, Georgopoulos D, Tsatsanis C (2014) Akt2 deficiency protects from acute lung injury via alternative macrophage activation and miR-146a induction in mice. J Immunol 192:394-406. doi: 10.4049/jimmunol.1300959

27. Hu L, Chen Z, Li L, Jiang Z, Zhu L (2019) Resveratrol decreases CD45(+) CD206(-) subtype macrophages in LPS-induced murine acute lung injury by SOCS3 signalling pathway. J Cell Mol Med 23:8101-8113. doi: $10.1111 / \mathrm{jcmm} .14680$

28. Pylayeva-Gupta Y (2016) Molecular Pathways: Interleukin-35 in Autoimmunity and Cancer. Clin Cancer Res 22:4973-4978. doi: 10.1158/1078-0432.CCR-16-0743

29. Du WX, He Y, Jiang HY, Ai Q, Yu JL (2016) Interleukin 35: A novel candidate biomarker to diagnose early onset sepsis in neonates. Clin Chim Acta 462:90-95. doi: 10.1016/j.cca.2016.09.005

30. Dasgupta S, Dasgupta S, Bandyopadhyay M (2020) Regulatory B cells in infection, inflammation, and autoimmunity. Cell Immunol 352:104076. doi: 10.1016/j.cellimm.2020.104076

31. Collison LW, Chaturvedi V, Henderson AL, Giacomin PR, Guy C, Bankoti J, Finkelstein D, Forbes K, Workman CJ, Brown SA, Rehg JE, Jones ML, Ni HT, Artis D, Turk MJ, Vignali DA (2010) IL-35mediated induction of a potent regulatory T cell population. Nat Immunol 11:1093-1101. doi: 10.1038/ni.1952

32. Held J, Preusse C, Doser A, Richter L, Heppner FL, Stenzel W (2013) Enhanced acute immune response in IL-12p35-/- mice is followed by accelerated distinct repair mechanisms in Staphylococcus aureus-induced murine brain abscess. J Infect Dis 208:749-760. doi: 10.1093/infdis/jit126

33. Cui H, Banerjee S, Guo S, Xie N, Ge J, Jiang D, Zornig M, Thannickal VJ, Liu G (2019) Long noncoding RNA Malat1 regulates differential activation of macrophages and response to lung injury. JCl Insight 4. doi: $10.1172 /$ jci.insight. 124522

34. Zhang Y, Huang T, Jiang L, Gao J, Yu D, Ge Y, Lin S (2019) MCP-induced protein 1 attenuates sepsisinduced acute lung injury by modulating macrophage polarization via the JNK/c-Myc pathway. Int Immunopharmacol 75:105741. doi: 10.1016/j.intimp.2019.105741

35. Ju M, Liu B, He H, Gu Z, Liu Y, Su Y, Zhu D, Cang J, Luo Z (2018) MicroRNA-27a alleviates LPSinduced acute lung injury in mice via inhibiting in fl ammation and apoptosis through modulating 


\section{Figures}

\section{Control group}

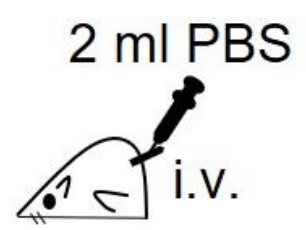

Saline

ALI group

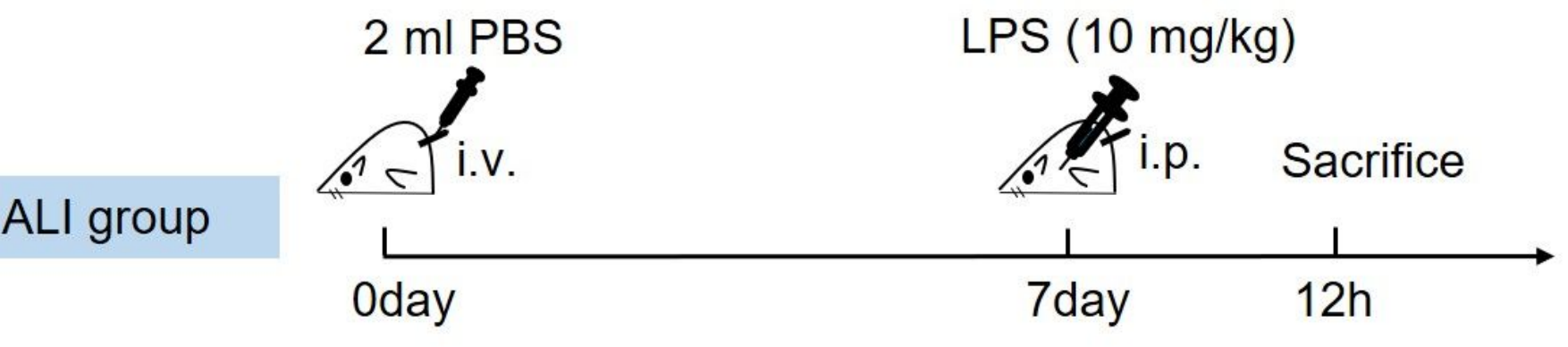

$\mathrm{ALI}+$ pcDNAIL35 group
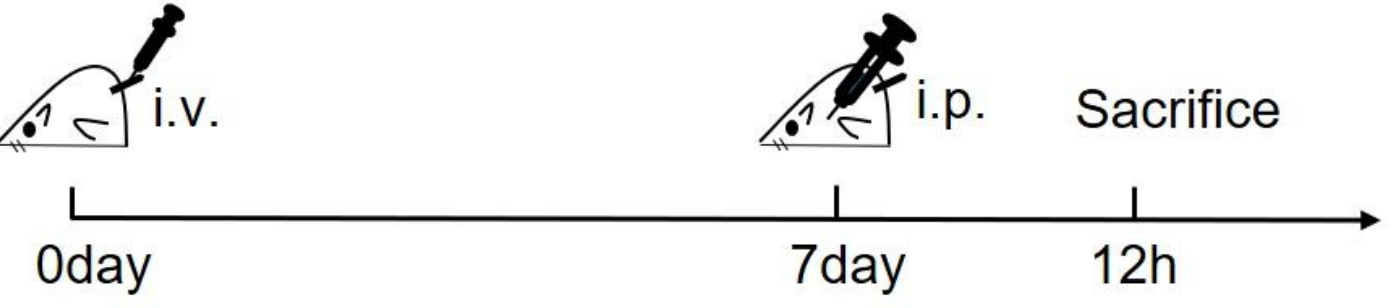

\section{$2 \mathrm{ml}$ PBS}

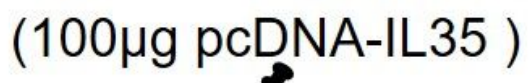

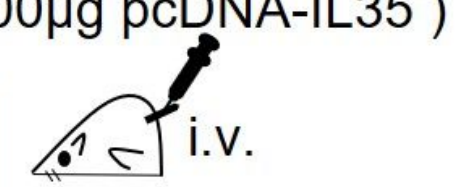

LPS $(10 \mathrm{mg} / \mathrm{kg})$

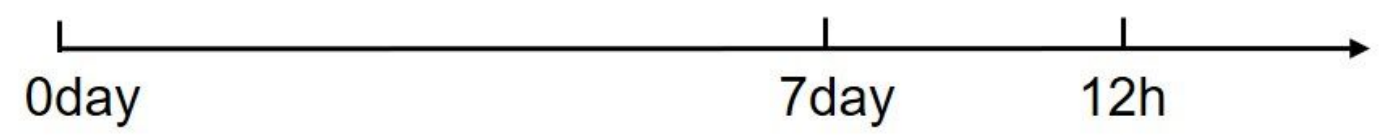

Figure 1

Experimental procedure.

Mice from the control group were given $2 \mathrm{ml}$ of PBS by i.v. and received an intraperitoneal injection (i.p.) of normal saline after 7 days. Mice from the ALI group were given $2 \mathrm{ml}$ of PBS by i.v. and received an i.p. injection of LPS ( $10 \mathrm{mg} / \mathrm{kg}$, Sigma, USA) after 7 days. Mice from the ALI+IL35 group were given $2 \mathrm{ml}$ of PBS containing $100 \mu \mathrm{g}$ pcDNA-IL35 by i.v. and received an i.p. injection of LPS ( $10 \mathrm{mg} / \mathrm{kg})$ after 7 days. 

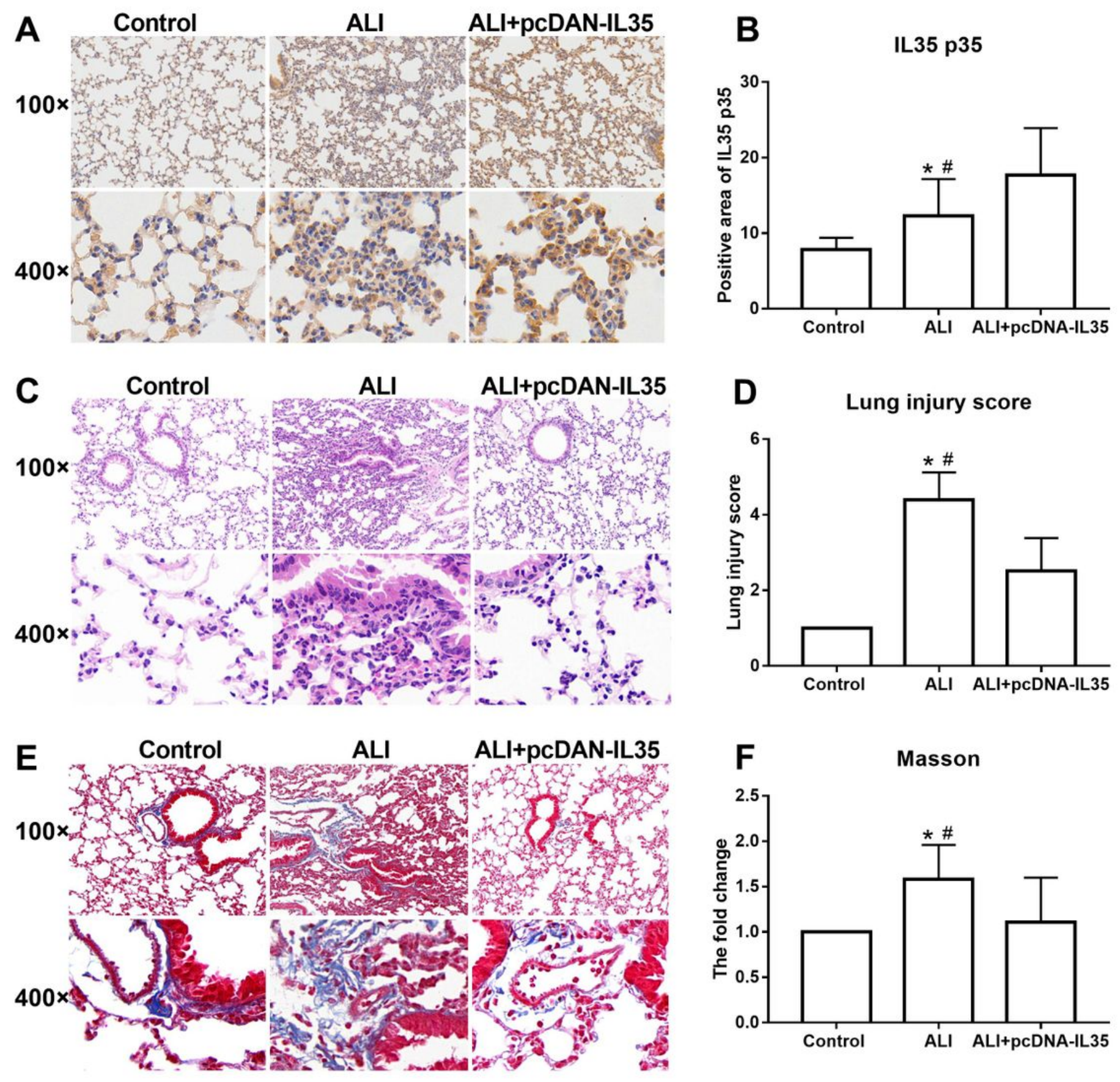

Figure 2

IL35 alleviates LPS-induced ALI in mice.

A Immunohistochemical staining for IL35 p35 in ALI in mice (original magnification $\times 10$ and $\times 40$ ). B Quantification of positive staining of IL35 p35 in ALI in mice. C Hematoxylin and eosin (HE) staining of lung tissues (original magnification $\times 10$ and $\times 40$ ). D Quantification of the lung injury score. E Masson staining of lung tissues (original magnification $\times 10$ and $\times 40$ ). F Quantification of collagen deposition. ${ }^{*} P$ $<0.05$ vs. Control. ${ }^{\#} P<0.05$ vs. ALI+pcDNA-IL35. 

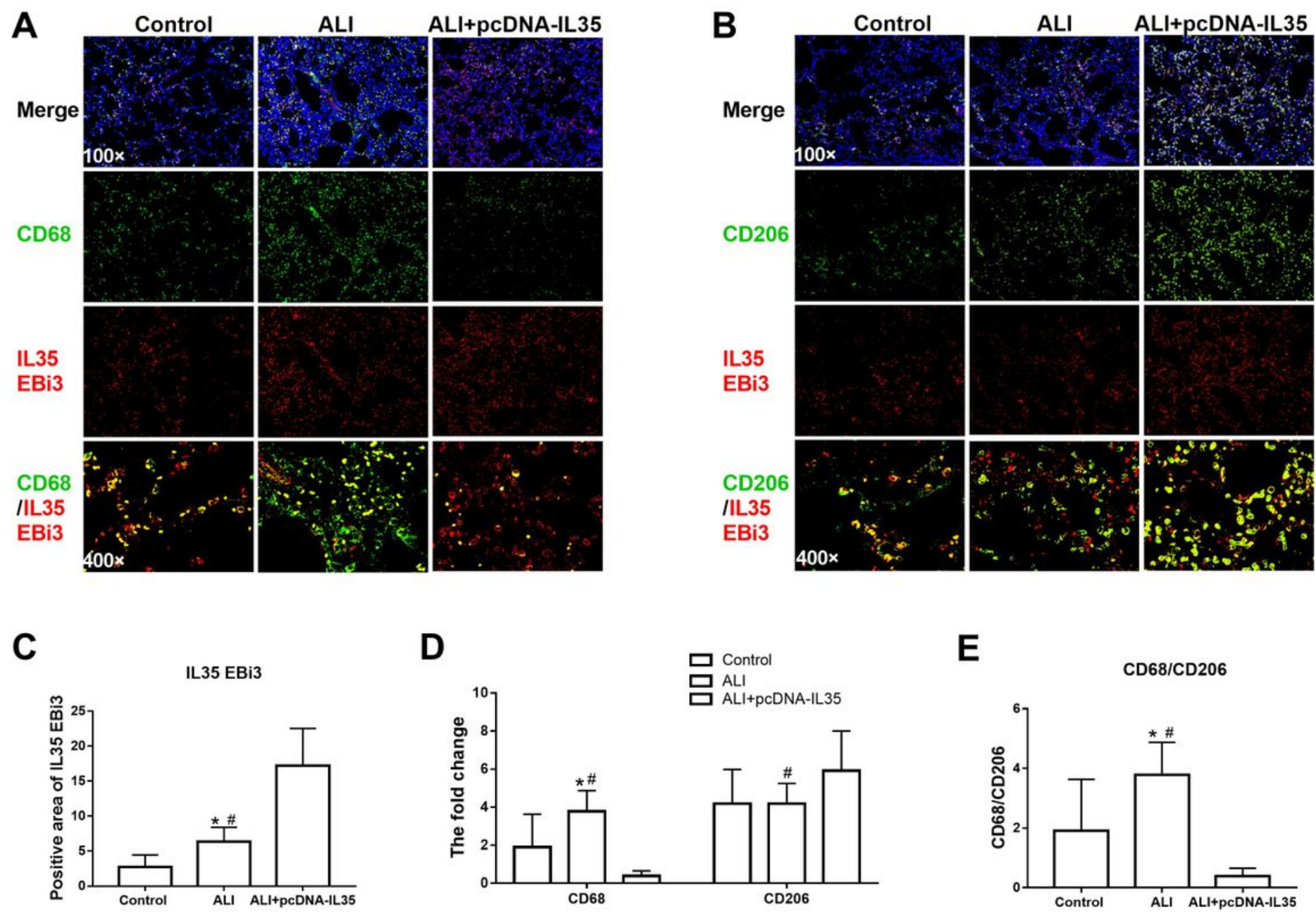

Figure 3

IL35 regulates macrophage polarization in ALI in mice.

A-B Immunofluorescence staining for IL35 EBi3 and CD68 or CD206 in ALI in mice (original magnification $\times 10$ and $\times 40$ ). C Quantification of positive staining for IL35 EBi3 in ALI in mice. D Quantification of positive staining for CD68 and CD206 in ALI in mice. E Quantification of the ratio of CD206/CD68 in ALI in mice. ${ }^{*} P<0.05$ vs. Control. ${ }^{\#} P<0.05$ vs. ALI+pcDNA-IL35. 


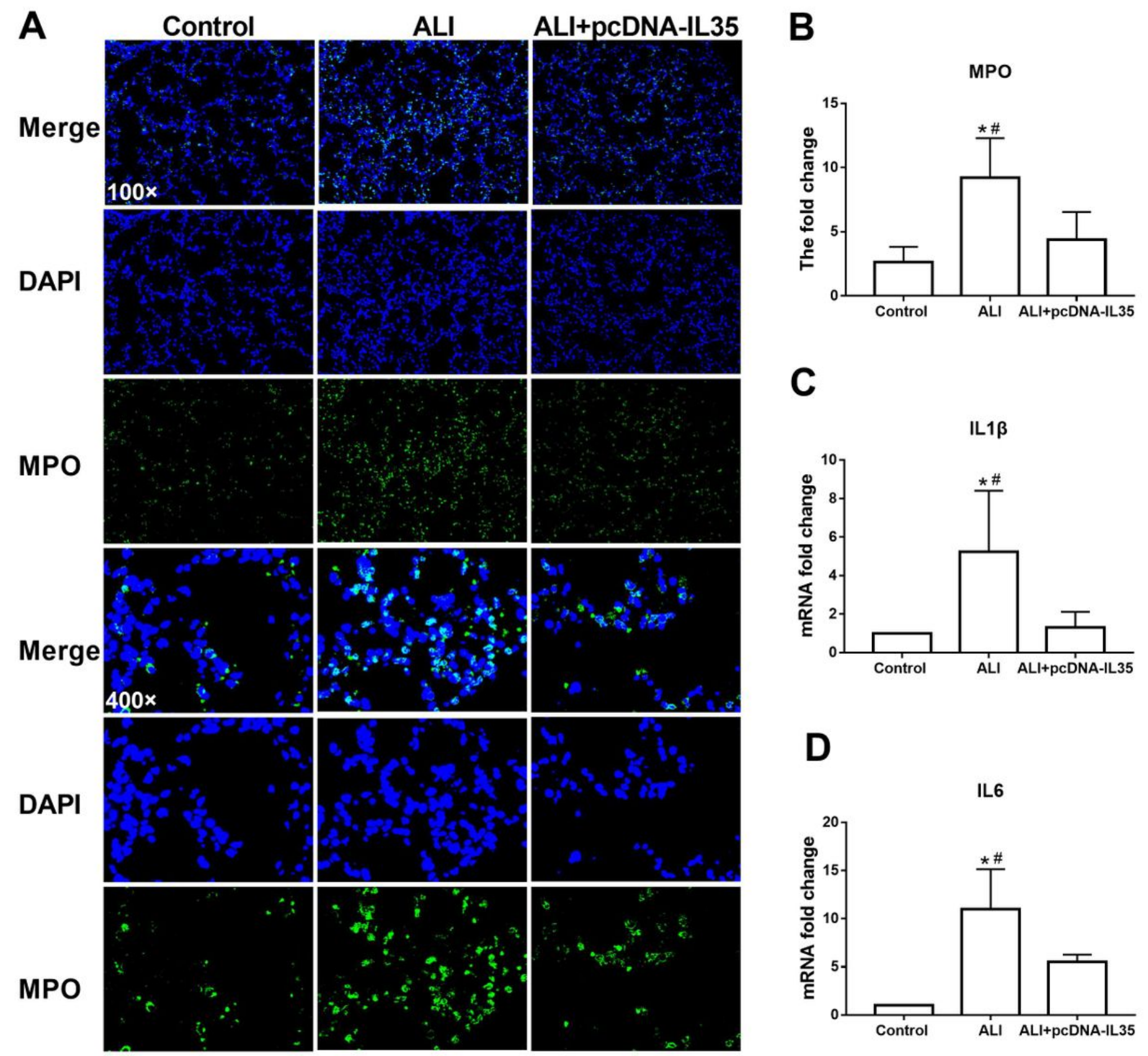

Figure 4

IL35 mitigates inflammation in ALI in mice.

A Immunofluorescence staining for MPO in ALI in mice (original magnification $\times 10$ and $\times 40$ ). B Quantification of positive staining for MPO in ALI in mice. C RT-PCR analysis for IL1 $\beta$. D RT-PCR analysis for IL6. ${ }^{*} P<0.05$ vs. Control. ${ }^{\#} P<0.05$ vs. ALI+pcDNA-IL35. 
A

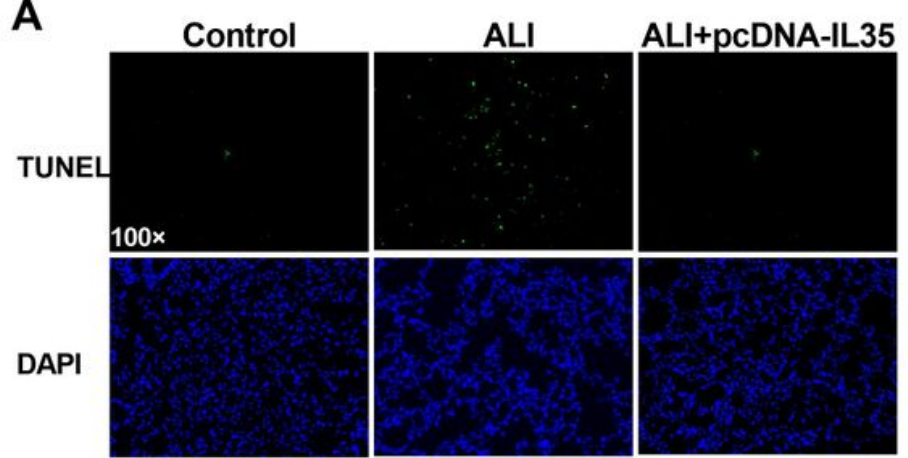

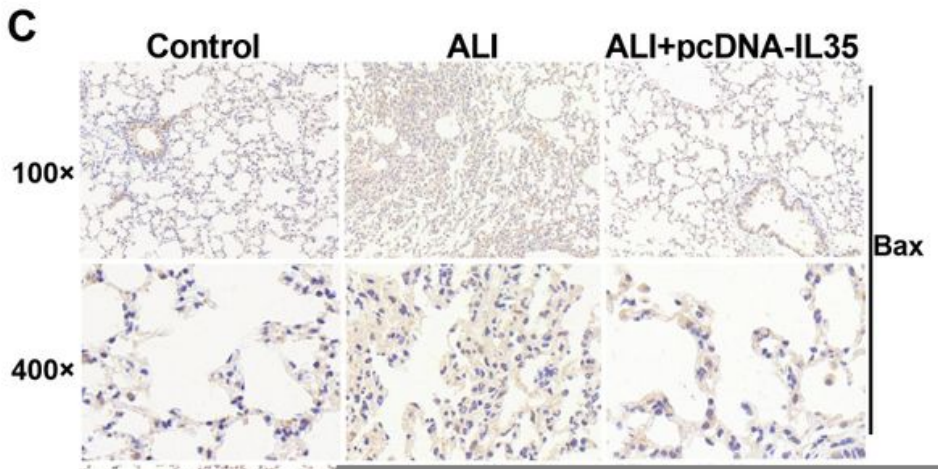

\section{Figure 5}

IL35 lessens apoptosis in ALI in mice.

A TUNEL staining of apoptotic cells (original magnification $\times 10$ and $\times 40$ ). B Quantification of apoptotic ALI cells in mice. C Immunohistochemical staining for $\mathrm{Bax}$ and $\mathrm{Bcl} 2$ in $\mathrm{ALI}$ in mice (original magnification $\times 10$ and $\times 40$ ). D Quantification of the ratio of Bax/Bcl2 in ALI in mice. ${ }^{*} P<0.05$ vs. Control. ${ }^{\#} P<0.05$ vs. ALI+pcDNA-IL35. 

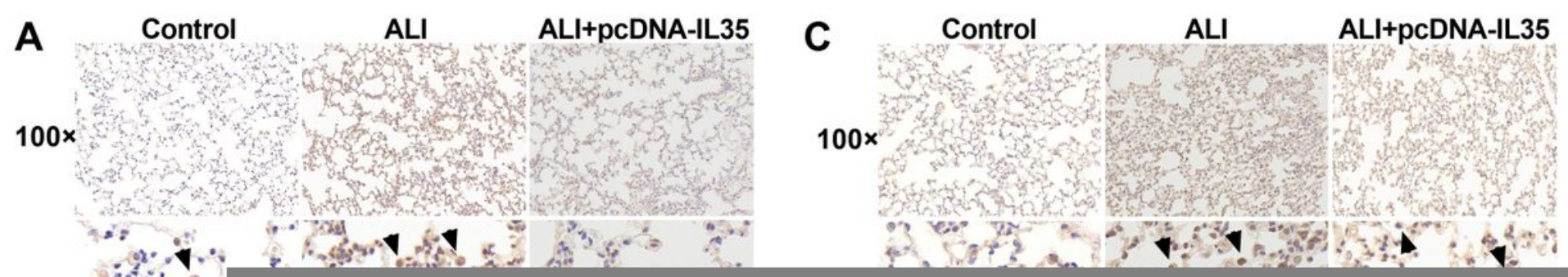

\section{Figure 6}

IL35 regulates TLR4/NFKB-P65 pathway.

A Immunohistochemical staining for TLR4 or MD2 in ALI in mice (original magnification $\times 10$ and $\times 40$ ). B Quantification of positive staining for TLR4 in ALI in mice. C Immunohistochemical staining for MD2 in $A L I$ in mice (original magnification $\times 10$ and $\times 40$ ). D Quantification of positive staining for MD2 in ALI in mice. E Immunohistochemical staining for p-P65 or P65 in ALI in mice (original magnification $\times 10$ and $\times 40)$. F Quantification of the ratio of p-P65/P65 in ALI in mice. ${ }^{*} P<0.05$ vs. Control. ${ }^{\#} P<0.05$ vs. ALI+pcDNA-IL35. 

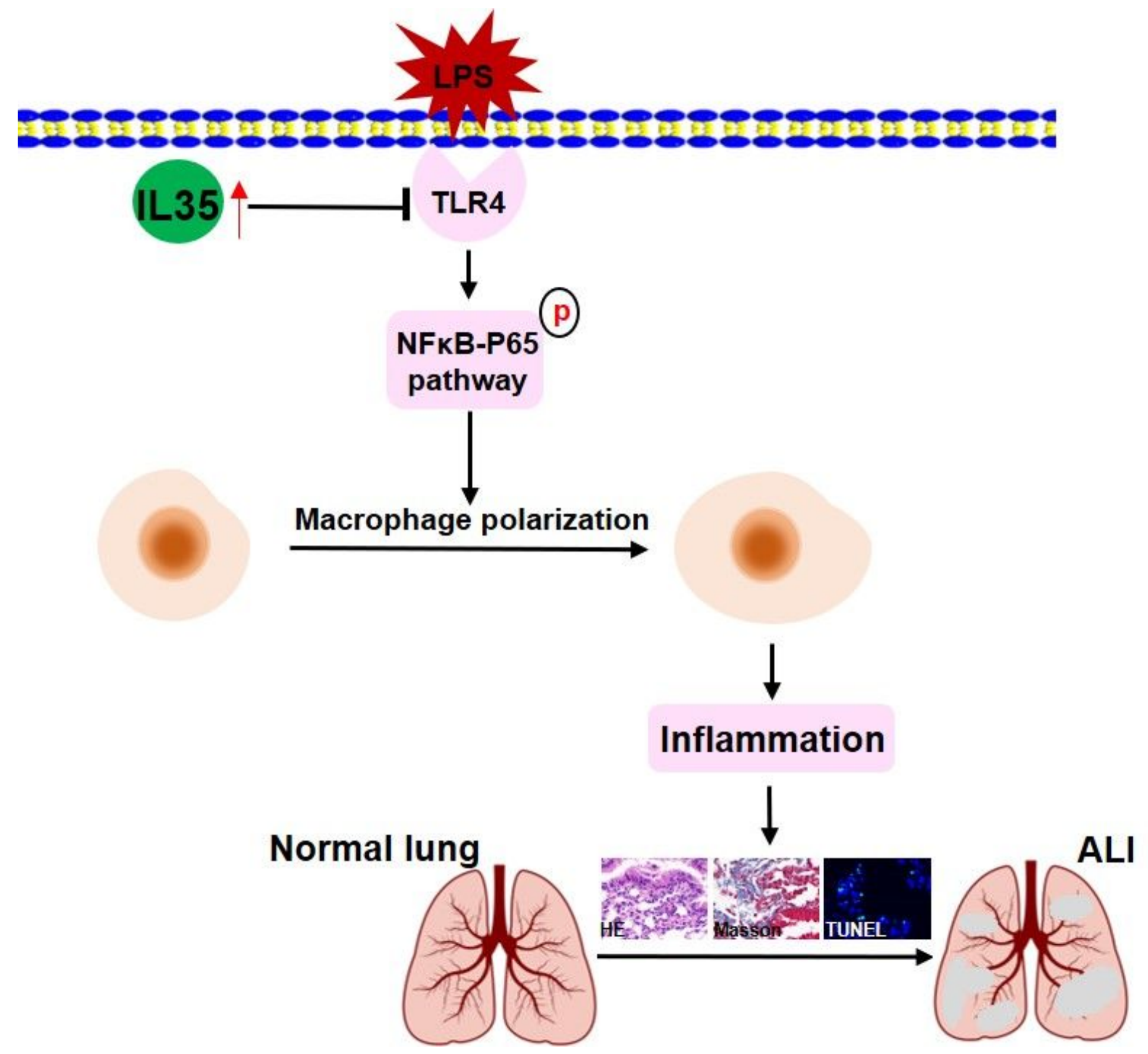

Figure 7

Schematic illustration of the role of IL35 in ALI.

IL35 relieved LPS-reduced inflammation and $A L I$ in mice by regulating M1/M2 macrophage polarization and inhibiting the activation of the TLR4/NFKB-P65 pathway. 